\title{
Management of Acute Variceal Bleed by Esophageal Variceal Ligation in an Unconventional Position-An Urban-Center Insight
}

\author{
Umesh Jalihal ${ }^{1}$ Prithvipriyadarshini Shivalingaiah ${ }^{2}$ Abhijith Bale,® \\ ${ }^{1}$ Karnataka Gastro Center, Bangalore, India \\ ${ }^{2} \mathrm{Al}$ Zahra Hospital Sharjah, Sharjah, United Arab Emirates

\begin{abstract}
Address for correspondence Umesh Jalihal, MBBS, MD, DM (Gastroenterology), DNB (Gastroenterology), Karnataka Gastro Center, Door no. 748, 5th Main, Between Modi And Nagapura Main Road, Mahalakshmi Puram, WCR, Bengaluru, Karnataka 560086, India (e-mail: drumeshj@yahoo.com).
\end{abstract}

\begin{abstract}
Keywords

- acute variceal bleed

- endoscopic variceal ligation

- cirrhosis

- endoscopy
\end{abstract}

Background Acute variceal bleed is a fatal complication in patients with liver cirrhosis, and it is important to achieve hemostasis at the earliest. Endoscopic variceal ligation (EVL) is a preferred modality in controlling variceal bleed. The blood pool in esophagus and oozing of blood from varices cause hindrance for variceal ligation leading to failure of therapy. In sitting position, it is hoped that visualization of esophagus may be better. With this background, EVL of patients in sitting position was done and results were analyzed. Methods Cirrhotics presenting with hematemesis and/or melena were included in the study. Clinical and endoscopic parameters were assessed. Among them, patients having hypotension and hepatic encephalopathy were excluded. Initial endoscopic assessment was done in left lateral position. In case of obscured visualization of esophagus due to flooding, if time taken to achieve hemostasis $>5$ minutes, position was changed to sitting from left lateral decubitus. Patients with isolated gastric variceal and ulcer bleed were further excluded. Results Among 78 acute bleed patients selected, 41 fulfilled the inclusion criteria for the study. During endoscopy, in 20 patients, EVL was done in left lateral decubitus position with head end elevation. Twenty-one patients were shifted from conventional left lateral position to sitting posture. Study revealed that patients undergoing EVL in sitting position had better visibility aiding the procedure and no aspiration during the procedure. There was no difference in the left lateral with head raised or without it. Duration of EVL was comparable to that of nonbleeders and additional sclerotherapy was not required in any case in both the groups. Following the procedure, patients were observed for 5 days. There was no failure of therapy which was analyzed according to Baveno VI consensus. However, there was delayed complication in one case due to sepsis and metabolic acidosis causing death.

Conclusion This study suggests, in acute variceal bleed cases, EVL in sitting position aids in better visibility and helps in achieving hemostasis early with least complications.

\section{Introduction}

Variceal bleeding is a leading cause of mortality and morbidity in patients with liver cirrhosis of various causes. ${ }^{1}$ Early mortality after an episode of acute variceal bleeding reaches $20 \%$. Despite the progress achieved over the past decades, the 6-week mortality associated with variceal bleeding is still in the order of 10 to $20 \%{ }^{2}$ Ligation of esophageal varices (EVs), proposed by G. V. Stiegmann in 1986, is currently the endoscopic method of choice for the treatment of variceal bleeding. Ligation of EVs obliterates them more rapidly and is accompanied by a smaller number of early recurrences of bleeding ${ }^{3}$ and according to Baveno VI consensus, it is the 
recommended form of endoscopic therapy for acute esophageal variceal bleeding. ${ }^{4}$

During an endoscopy due to flooding of field by active bleed, it becomes technically challenging for band ligation and leads to failure of treatment or use of rescue therapies thereafter and according to the Asian Pacific Association for the Study of the Liver guidelines rebleed within 24 hours of endoscopic therapy leads to increased morbidity and mortality. ${ }^{5}$ With this background, the study was designed to aid in better outcome of endoscopic variceal ligation (EVL) in acute variceal bleed scenario.

\section{Materials and Methods}

From November 2016 to August 2019, all patients aged between 18 and 75 years presenting to emergency or referred from other centers to our tertiary center with history of hematemesis were analyzed. Institutional ethics committee permission and patient consent with special emphasis on the sitting posture if necessary were obtained. Onsets of hematemesis and/or melena within 48 hours of reporting to hospital were selected, and the detailed history and examination suggesting chronic liver disease or portal hypertension were included. These included history of chronic alcohol intake, presence of jaundice, ascites, splenomegaly, palpable firm left hepatic lobe, presence of abdominal wall collaterals, pedal edema, gynecomastia, testicular atrophy, parotid enlargement, vascular spiders, leukonychia, and palmar erythema and known cirrhotics with a previous history of variceal bleed or previous endoscopy showing EVs.

The physical examination, while complete, was directed at findings relevant to gastrointestinal bleeding. Resting tachycardia with pulse rate $>100 / \mathrm{min}$, orthostatic hypotension defined as a decrease in the systolic blood pressure of more than $20 \mathrm{~mm} \mathrm{Hg}$ or an increase in the pulse of more than 20 beats/min from recumbency to standing and hypotension with systolic blood pressure $<90 \mathrm{~mm} \mathrm{Hg}$ and patients in shock with thready, weak pulse, in spite of intravenous (IV) fluids and initial resuscitative measures were excluded from the study. Patients with features suggestive of hepatic encephalopathy, respiratory distress with $\mathrm{SpO}_{2}<90 \%$ on room air, respiratory rate $>20 /$ min or requiring endotracheal intubation, orthopaedic problems or cerebrovascular accidents which hinder the patient to achieve a sitting posture, uncooperative patients, or those who refused to give consent were also excluded from the study.

Patients were kept nil orally, and circulating blood volume was restored with fluids and blood transfusions if hemoglobin was less than $8 \mathrm{~g} / \mathrm{dL}$. IV ceftriaxone $1 \mathrm{~g}$ was given to all patients before shifting for endoscopy.

Door-to-endoscopy time was kept at a maximum of 12 hours. Initial endoscopy was done in conventional left lateral decubitus position with slight head raised, using Olympus GIF-H180 videoendoscope after local Xylocaine spray in the throat.

\section{Inclusion Criteria}

1. Acute variceal bleed with obscured visibility

2. Inability to achieve hemostasis in 5 minutes in left lateral decubitus.

\section{Exclusion Criteria}

1. Hypotension

2. Hepatic encephalopathy $>\mathrm{Gr}$ II

3. Systemic conditions precluding sitting posture

4. Isolated gastric varices (GVs)

5. Mallory-Weiss tear or ulcers.

No IV sedation or anesthesia was used. Esophageal variceal hemorrhage was identified as bleeding from EVs confirmed at endoscopy and flooding of visual field in esophagus with spurting or actively oozing was selected for the second step of the study.

The selected patients were made to switch the position to sitting ( - Fig. 1) and scope was fitted with multishot band ligator-Omniview (Medelec Systems Pvt. Ltd.) and introduced into the esophagus. Bands were applied starting just above the esophagogastric junction in a spiral fashion until the bleed stopped or obliteration of varices was achieved, with a maximum of six bands.

Patients were monitored in the observation room for 12 hours, hematocrit was checked every 8 hours, and hemoglobin was checked every 12 hours. They were then shifted to the ward, started on liquids orally, and observed for a total of 5 days from the procedure, with hemoglobin and hematocrit checked once daily.

Transfusion target was hemoglobin of $8 \mathrm{~g} / \mathrm{dL}$ or hematocrit of $24 \%$. They were discharged if there was no further adverse outcome or failure of therapy defined using Baveno VI consensus as:

1. Fresh hematemesis in 2 hours after therapeutic endoscopy, $>100 \mathrm{~mL}$ of fresh blood

2. Drop in hemoglobin $>3 \mathrm{~g}$ and hematocrit of $>9 \%$ with no blood transfusion

3. Death.

\section{Results}

In this study, 78 patients fulfilled both inclusion and exclusion criteria. Causes other than variceal bleed were noted in 10 patients which included Mallory-Weiss tear, duodenal ulcer, gastric ulcer, ulceroproliferative growth, and post-EVL ulcer bleed. Five patients had isolated GVs and esophagogastric varices (-Fig. 2). Twenty-two patients with EVs had minimal or no active bleed in esophagus, presenting with either stigmata of recent bleed such as red signs, platelet plugs, or pooling of blood in stomach. Forty-one patients had esophageal variceal bleed with minimal or no visualization due to active bleed in esophagus and underwent EVL in sitting position. In 20 patients, EVL was done in left lateral decubitus with slight head end 


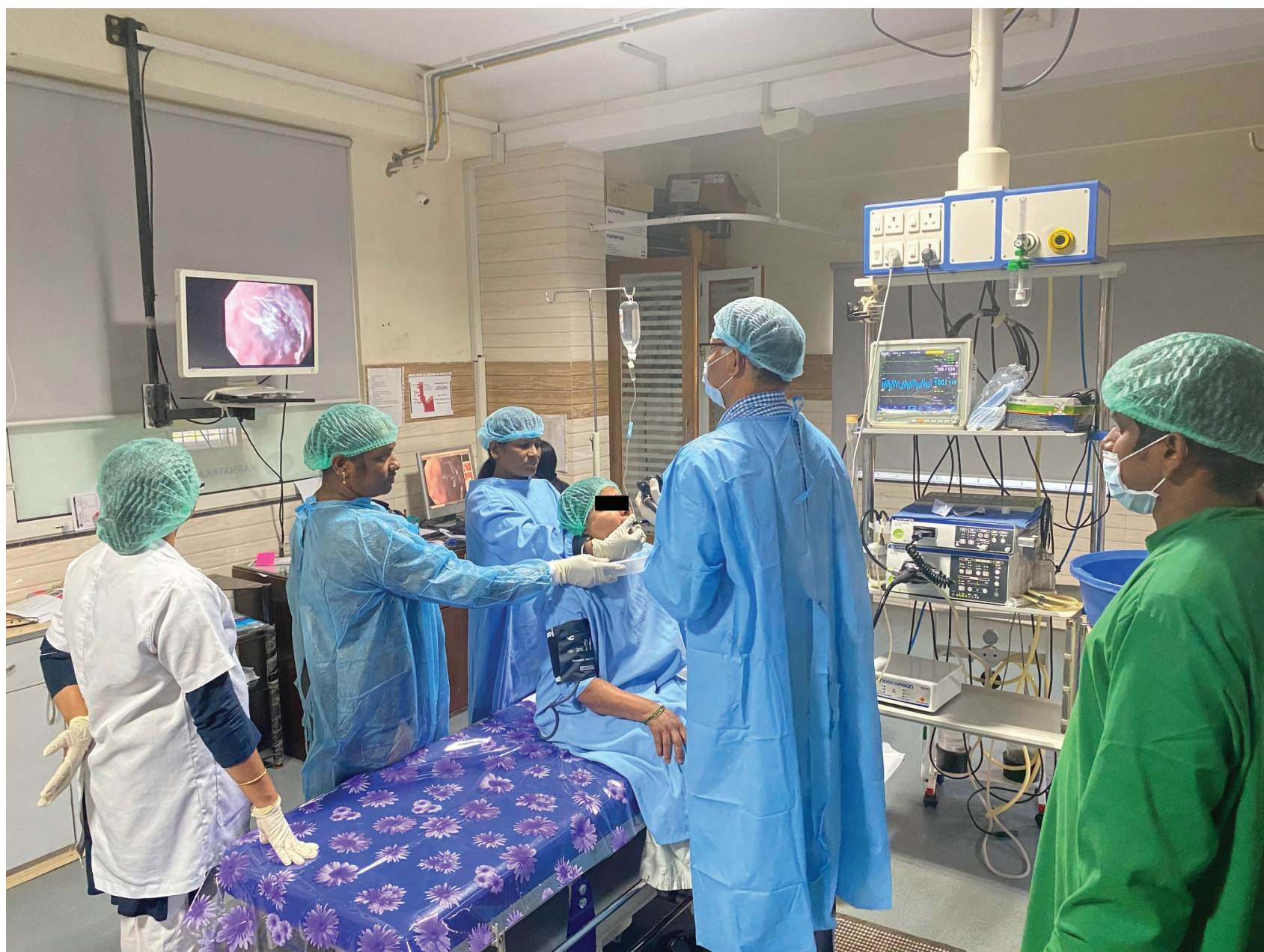

Fig. 1 EVL procedure in sitting position.

elevation and in 21 patients, EVL was done in sitting position ( - Fig. 1 ).

Baseline characteristics of 41 patients were as follows:

- Mean age (38-72) 56.6 years in first group in left lateral decubitus with head raise and 54 years in second group with banding in sitting position.

- Nineteen males and one female in first group, and 19 males and 2 females in second group. Six patients were known cirrhotics who had undergone EVL previously, three of them had emergency EVL previously. Alcohol was noted as cause of cirrhosis in 34 patients, hepatitis B in 2 patients, and nonalcoholic fatty liver disease in 5 patients. Four patients were Child-Turcotte-Pugh (CTP)-A, 19 patients were CTP-B, and 18 patients were CTP-C, divided equally into two groups (-Table 1).

- Mean time between onset of hematemesis and/or melena to presenting in the hospital was 23.8 hours (4-38 hours). Mean heart rate was 90/min (80-98/min). Mean systolic blood pressure was $100 \mathrm{~mm} \mathrm{Hg}$, mean hemoglobin was $9 \mathrm{~g} / \mathrm{dL}$, and mean hematocrit was $26 \%$.

\section{Procedure-Related Results}

Mean time between arrival to hospital and endoscopy was 7.8 hours.

On changing the position of the patient, the blood gravitated to the stomach thereby aiding better visibility (-Figs. 3-5). Bands were applied ranging from four to six in number in both groups. The time taken for the ligation during sitting position was comparable to the prophylactic banding with no delays due to obscurity of vision or technical difficulties. There was more time taken during band ligation in lateral decubitus position. There was no case of aspiration in patients of both the groups during the procedure. There was no requirement for additional sclerotherapy in all cases in both groups.

Postprocedure: There was no significant rebleed and fall in hemoglobin or hematocrit in any cases. Blood transfusions required ranged from 0 to 2 transfusions in total. Although there was one death in the first group, after 38 hours of procedure, cause of death was attributed to metabolic acidosis and septicemia. 


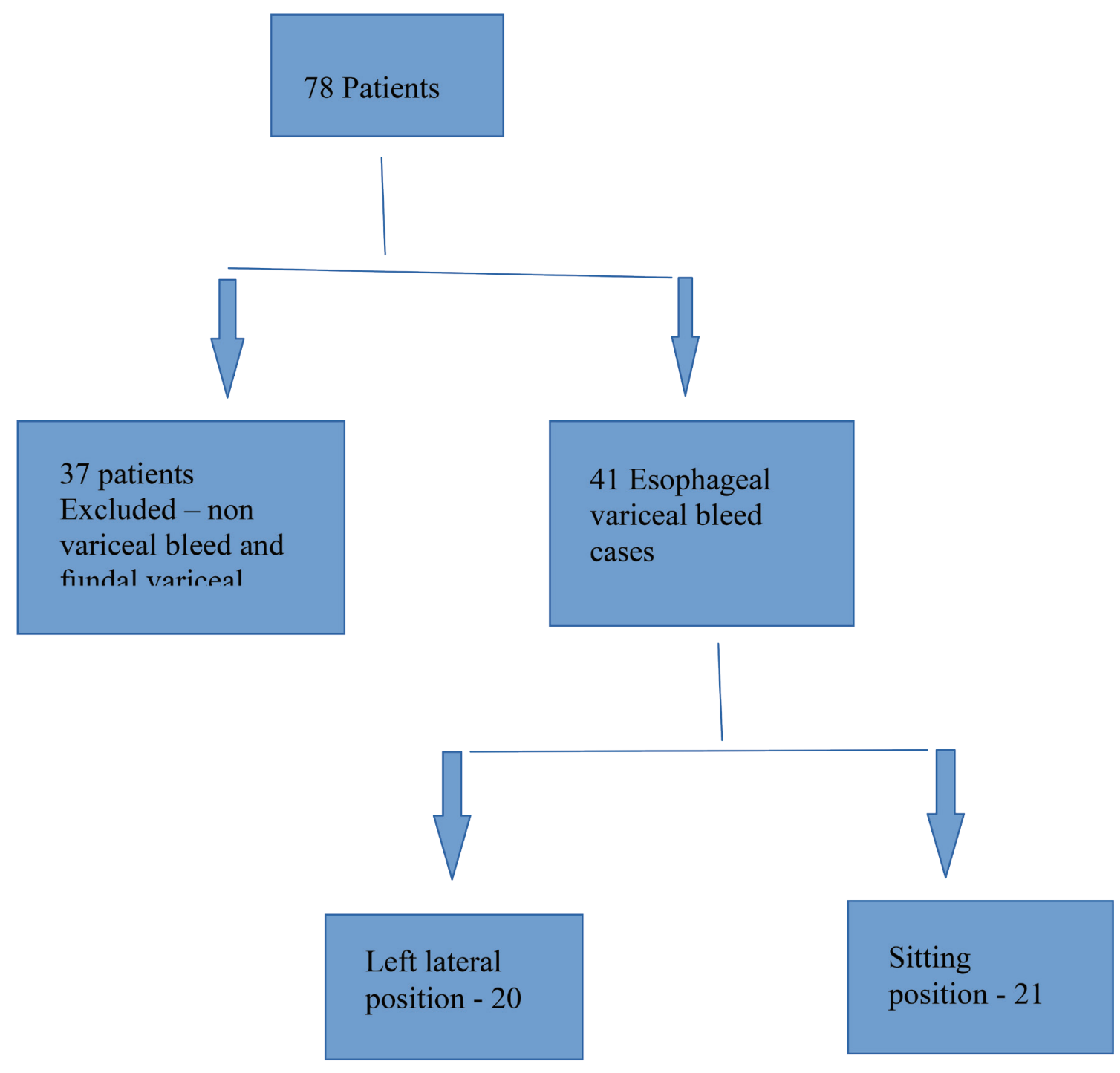

Fig. 2 Patient flow and distribution.

Table 1 Baseline characteristics and procedure-related parameters

\begin{tabular}{|l|l|l|}
\hline Parameters & $\begin{array}{l}\text { Lateral } \\
\text { decubitus-EVL }\end{array}$ & Sitting-EVL \\
\hline Age (mean) & 56.6 & 54 \\
\hline Male & 19 & 19 \\
\hline Female & 1 & 2 \\
\hline CTP-A and -B & 12 & 11 \\
\hline CTP-C & 8 & 10 \\
\hline $\begin{array}{l}\text { EVL duration } \\
\text { (mean) }\end{array}$ & 5.2 min & 4.8 min \\
\hline $\begin{array}{l}\text { SpO } \\
\text { (\%) }\end{array}$ & 96 & 95 \\
\hline $\begin{array}{l}\text { Mean arterial pres- } \\
\text { sure (mm Hg) }\end{array}$ & 86 & 84 \\
\hline
\end{tabular}

Abbreviations:EVL, endoscopic varicealligation; CTP, Child-Turcotte-Pugh.

On 6 weeks follow-up, 14 patients had no hematemesis or melena and there was no further drop in hemoglobin and hematocrit values in both groups. It was noted that patients undergoing EVL in sitting position had better endoscopic visibility and required lesser procedure time.

\section{Discussion}

Variceal bleeding constitutes 70\% of all upper gastrointestinal bleeding episodes in patients with portal hypertension, and they result from EVs, GVs, or ectopic varices. Remaining $30 \%$ is due to other causes, such as portal hypertensive vasculopathies, Mallory-Weiss lesions, and ulcers. ${ }^{6,7}$ In this study, among initially selected patients, $19.2 \%$ had nonvariceal etiology and were excluded ( - Fig. 2). Nearly $50 \%$ of patients with newly diagnosed liver cirrhosis have accompanying varices; de novo appearance of varices or worsening of preexisting varices is noted in $7 \%$ of the cirrhotic patients yearly. Index bleeding episode occurs in $12 \%$ of patients yearly. The factors predicting the risk of variceal bleed include the size of the varix, red color signs, and the degree of deterioration of liver function. ${ }^{8}$

Significance of active bleeding (spurting or oozing) at endoscopy has evolved from Baveno I, the consensus was that active bleeding at endoscopy is a predictor of failure to control bleeding, while its prognostic value for mortality was unclear. ${ }^{6}$ Recent consensus says the presence of active bleeding at endoscopy predicts early rebleeding. ${ }^{5}$ The time 


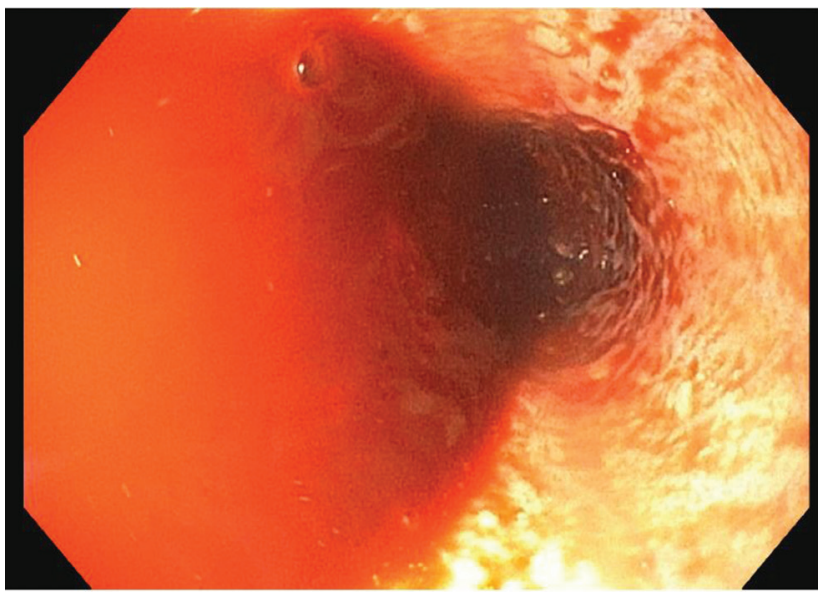

Fig. 3 Blood flood in lower esophagus in left lateral decubitus.

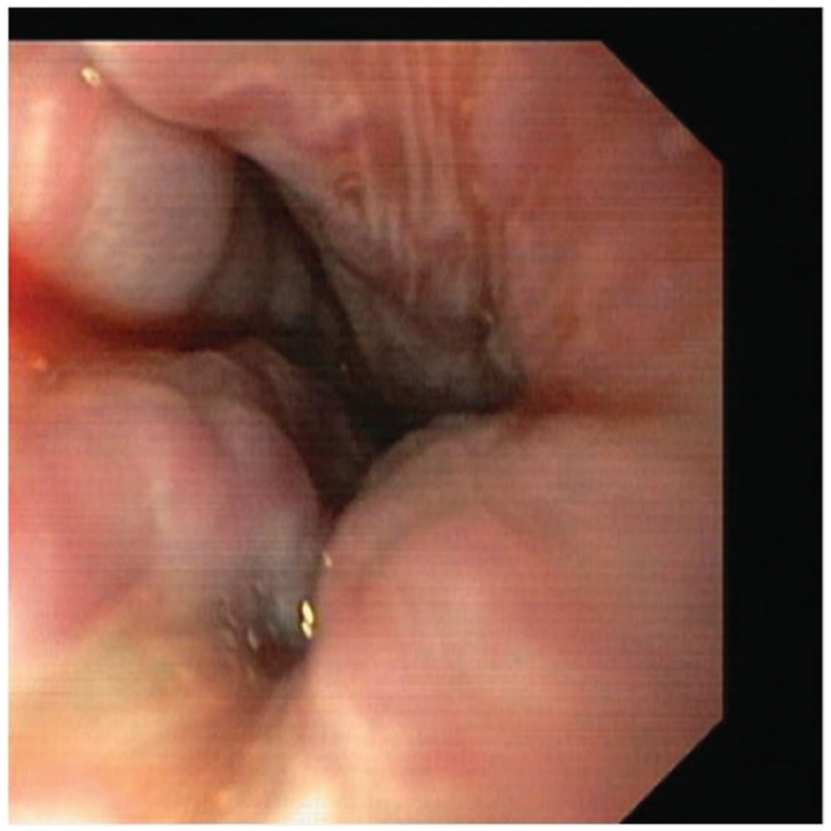

Fig. 4 Esophageal Varices unmasked in sitting position.

frame for rebleeding as defined in Baveno III was 48 hours. However, it was increased to 120 hours in Baveno IV. In this study, onset of bleed within 48 hours and presence of active bleed on endoscopy were selected and followed up for 120 hours following the procedure.

In a study of 210 patients with acute variceal bleeding and hemodynamic stability, performing endoscopic treatment at 4,8 , and 12 hours after arriving at the hospital did not significantly affect the mortality rate. ${ }^{9}$ However, in another study, performing endoscopic therapy after more than 15 hours of hospital arrival significantly increased the mortality rate. ${ }^{10}$ It is recommended that endoscopic treatment be performed as soon as possible, that is, within 12 hours, in patients with variceal bleeding. ${ }^{10}$ In this study, arrival to endoscopy time ranged between 4 and 11.5 hours (mean of 7.8 hours). Patients with conditions precluding urgent endoscopy such as hypovolemic or septicemic shock and hepatic encephalopathy were excluded, thereby allowing early endoscopy.

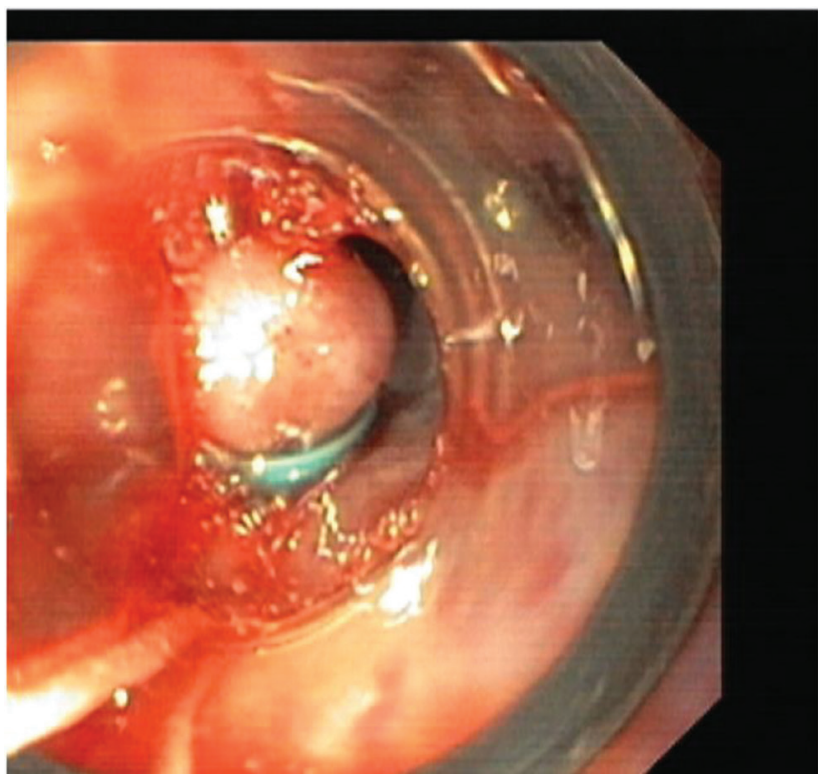

Fig. 5 Banded varix on sitting position.

In patients with decompensated liver cirrhosis accompanied by ascites or hepatic encephalopathy, acute variceal bleeding is associated with a high mortality rate. ${ }^{11}$ Mortality in this study was noted in one patient with decompensated liver cirrhosis and sepsis. Orthostatic hypotension suggests a loss of $15 \%$ or more of the blood volume. Hypotension is associated with a $30 \%$ loss of blood volume. ${ }^{12}$ Excluding these critical patients may explain lesser morbidity and mortality in this study.

Further, an observation was made during the study that sitting position makes it technically better for the endoscopist in acute variceal bleed and decreases the EVL time. There were no adverse outcome in lateral decubitus with head elevation, but the difficulty in banding due to poor visibility persisted. This study suggests that unconventional position of making the patient sit while performing EVL can be considered compared with left lateral decubitus position to improve outcome in acute variceal bleed scenario.

\section{Conflict of Interest}

None declared.

\section{References}

1 Gado AS, Ebeid BA, Abdelmohsen AM, Axon AT. Clinical outcome of acute upper gastrointestinal hemorrhage among patients admitted to a government hospital in Egypt. Saudi J Gastroenterol 2012;18(1):34-39

2 de Franchis R; Baveno V Faculty. Revising consensus in portal hypertension: report of the Baveno $\mathrm{V}$ consensus workshop on methodology of diagnosis and therapy in portal hypertension. J Hepatol 2010;53(4):762-768

3 Dai C, Liu WX, Jiang M, Sun MJ. Endoscopic variceal ligation compared with endoscopic injection sclerotherapy for treatment of esophageal variceal hemorrhage: a meta-analysis. World J Gastroenterol 2015;21(8):2534-2541

4 de Franchis R, Abraldes JG, Bajaj J, et al; Baveno VI Faculty. Expanding consensus in portal hypertension: Report of the Baveno VI Consensus Workshop: stratifying risk and 
individualizing care for portal hypertension. J Hepatol 2015;63(3):743-752

5 Sarin SK, Kumar A, Angus PW, et al; Asian Pacific Association for the Study of the Liver (APASL) Working Party on Portal Hypertension. Diagnosis and management of acute variceal bleeding: Asian Pacific Association for Study of the Liver recommendations. Hepatol Int 2011;5(2):607-624

6 D’Amico G, De Franchis R; Cooperative Study Group. Upper digestive bleeding in cirrhosis. Post-therapeutic outcome and prognostic indicators. Hepatology 2003;38(3):599-612

7 Terés J, Bordas JM, Bru C, Diaz F, Bruguera M, Rodes J. Upper gastrointestinal bleeding in cirrhosis: clinical and endoscopic correlations. Gut 1976;17(1):37-40

8 Brocchi E, Caletti G, Brambilla G, et al; North Italian Endoscopic Club for the Study and Treatment of Esophageal Varices. Prediction of the first variceal hemorrhage in patients with cirrhosis of the liver and esophageal varices. A prospective multicenter study. N Engl J Med 1988;319(15):983-989

9 Cheung J, Soo I, Bastiampillai R, Zhu Q, Ma M. Urgent vs. non-urgent endoscopy in stable acute variceal bleeding. Am J Gastroenterol 2009;104(5):1125-1129

10 Hsu YC, Chen CC, Wang HP. Endoscopy timing in acute variceal hemorrhage: perhaps not the sooner the better, but delay not justified. Am J Gastroenterol 2009;104(10):2629-2630, author reply 2630-2631

11 Toubia N, Sanyal AJ. Portal hypertension and variceal hemorrhage. Med Clin North Am 2008;92(3):551-574, viii

12 Bonanno FG. Hemorrhagic shock: the "physiology approach". J Emerg Trauma Shock 2012;5(4):285-295 